\title{
A Methodology to Improve Cash Demand Forecasting for ATM Network
}

\author{
Saad M. Darwish
}

\begin{abstract}
Developing cash demand forecasting model for ATM network is a challenging task as the chronological cash demand for every ATM fluctuates with time and often superimposed with non-stationary behavior of users. In order to improve the forecasting precision of ATM cash demand, an Interval Type-2 Fuzzy Neural Network (IT2FNN) has been utilized in this paper. The antecedent parts in each rule of the IT2FNN are interval type-2 fuzzy sets in view of conditions regarding time, location, cash residual and other factors that could lead to consider cash upload able to keep cash at the right levels to meet user demand. The employed IT2FNN has both on-line structure and parameter learning abilities. Simulation results for ATM cash forecasting show the feasibility and effectiveness of the proposed method.
\end{abstract}

Index Terms-Electronic services optimization, Fuzzy logic, Cash demand forecasting, ATM.

\section{INTRODUCTION}

ATM (Automatic Teller Machine) is a computerized telecommunication device that provides financial transactions in a public space without the need for the human clerk [1]. Optimal cash management and services availability is one of the most important factors in the ATM network services business. Using cash management optimization and efficient cash loads routing, banks can avoid of stuck ATMs with cash and manage the system in dynamically changing environment by achievement the different requirements of ATM network participants. Recently, more banks are turning their attention to derive greater efficiency in how they manage their cash at ATMs [2].

Effective cash management starts with an automated solution that uses sophisticated algorithms to accurately predict currency supply and demand, allowing banks to forecast demand and proactively manage currency throughout their network plus with reducing of currency transportation and servicing costs. These algorithms should also be flexible enough to allow the bank to re-forecast future demand, perform what-if analyses and optimize the network as the cash distribution environment evolves.

The key to the ATM's forecasting algorithms is to capture and process the historical data such that it provides insight into the future. Newly, some authors attempted to optimize the cash by modeling and forecasting the demand [2]-[4]. However, the high variance and non-stationary of the underlying stochastic cash demand process can affect

Manuscript received November 14, 2012; revised February 22, 2013.

S. M. Darwish is with the Department of Information Technology, Institute of Graduate Studies and Research, Alexandria University, 163 Horreya Avenue, El Shatby 21526, P.O. Box 832, Alexandria, Egypt ( e-mail :saad.darwish@alex-igsr.edu.eg) reliability of such approaches. Furthermore, the demand of cash is not only influenced by time, but it follows different tendencies that make modeling even more difficult. For example, how holidays affect the use of ATM depends on where the teller is located.

This research proposes rule-based ATM cash demand forecasting method as an applied alternative to time series-based cash management. Time series method has smaller calculation, faster speed and largest application range, but the forecasting accuracy cannot guarantee to meet cash needs and it has no adaptive learning capabilities. In other words, this work could be interested in finding a set of rules, which take the withdrawal affecting input patterns of a week and that if applied to current operation of ATMs can lead to the decision of when and how upload the machine. The proposed rule-based method combines the merits of neural network, provide a methodology for solving many types of nonlinear problems that are difficult to solve by traditional techniques, and type-2 fuzzy logic controller (T2FLC) that has been shown to be a powerful paradigm to handle the high level of uncertainties in real-world applications [5].

The remaining of this paper is organized as follows: Section 2 briefly overviews exiting ATM cash management techniques. Section 3 formally defines the problem at hand and the generative based solution. Section 4 describes the proposed rule-based forecasting model. The experimental results and a short discussion are given in Section 5. Finally, concluding remarks are derived in the last section, which also highlights direction for future work.

\section{EXISTING METHODS FOR CASH FORECASTING}

In the literatures, techniques used for cash demand forecasting can be broadly classified into four groups [5-8]: (1) Time series method that predicts future cash need based on the past values of variable and/or past errors. Generally, techniques in time series approach work well unless there is an abrupt change in the environment or variables that are believed to affect the cash withdrawal pattern. (2) Factor analysis method, which is based on the determination of various factors that influence the cash demand pattern and calculating their correlation with actual cash withdrawal. The purpose is to determine the functional form of this influence (independent variables) and to use this to forecast future values of the dependent variables. However, estimating this functional form is more difficult when they have a non-linear relationship. (3) Fuzzy expert system approach that tries to imitate the reasoning of a human operator. The idea is to reduce the analogical thinking behind the intuitive 
forecasting to formal steps of logic. However, expert systems have been found to be feasible only when narrowly confined. In addition, there are many difficulties to incorporate adequate expert knowledge into the rules of fuzzy system. (4) Neural networks approach that maps the relationships between various factors affecting the cash withdrawal and the actual cash withdrawal. The evaluation of this cash forecasting system has been done on the basis of standard statistical measures like percentage errors. Artificial neural networks method has better robustness; nonlinear mapping ability and strong self-learning ability, but it has slow convergence and probably converges to a local minimum point.

Some drawbacks can be identified in the previous commercially available solutions. For instance, techniques based on parameters are generally static and do not adapt during operation, so that on-line cash optimization is not made possible. Other techniques are largely depending on linear regression models with seasonality coefficients customized for every ATM. The development of such models is relatively complicated and differs for various ATM.

\section{PRoblem Statement AND Solution}

In ATM, while it would be devastating to run out of cash, it is important to keep cash at the right levels to meet customer demand. In such case, it becomes very necessary to have a forecasting system in order to get a clear picture of demands in advance. Having the models to forecast the daily (or weekly) cash demand for every ATM, it is possible to plan and to optimize the cash loads for the whole ATM network.

In general, cash withdrawal is a stochastic process whose characterization tends to change over time due to several factors that have a strong uncertainty. Considering this obstacle and to eliminate the existing techniques drawbacks, the work presented in this paper proposes a new forecasting method based on IT2FNN. This method searches for a set of rules in which the cash upload decision could be made upon conditions regarding withdrawal affecting factors with the aim to facilitate the forecasting operation for whole ATM network.

Although there are several examples on the applications of neural networks to the problem of cash forecasting, no work has been reported on the using of IT2FNN for ATM cash forecasting. This method not only improves the overall performance of the forecasting system but also adapts to the dynamic nature for ATM cash demand. The functional principle of the proposed method is discussed below.

Problem Formulation and Methodology

The problem of rule-based cash demand forecasting can be formulated by IF-THEN rules as follow:

$$
\begin{array}{r}
\mathbf{I F} c_{1}\left(A T M_{i}, t\right)=a_{1} \text { AND.... } \\
\text { AND } c_{n}\left(A T M_{i}, t\right)=a_{n} \\
\text { THEN UPLOAD } l_{i}
\end{array}
$$

meaning that if the ATM number $i$ at time $t$ meets conditions $c_{1}, c_{2}, \ldots .$. , and $c_{n}$ then this ATM is uploaded with $l$ cash level. Herein, the antecedent parts in each rule are conditions on some factors (e.g. time, cash level, location, etc.) and the consequent part are a set of possible recharges to apply when premises are met. In formal, given a set of premises, rule specifications, $R$, belong to the space:

$$
K \subseteq R \equiv C_{1} \times C_{2} \times \ldots \ldots \times C_{n} \times L
$$

where $C_{\mathrm{i}}$ is the set of possible outcomes of antecedent and $L$ is the set of possible recharge levels. In this case, the aim is to find the subset of $K$ that when applied to a distinct ATM or to a group of them, minimizes the daily average ATM cash stock $S$ within the time interval $T$ that is [9]:

$$
\begin{gathered}
K=\underset{\substack{K \subseteq R \\
\arg \min }}{ } S_{K}(T) \\
S_{k}(T)=\sum_{A T M \in A} S\left(A T M_{i}, T\right), A \subseteq A T M s \\
S\left(A T M_{i}, t\right)=S_{0}\left(A T M_{i}\right)+\frac{1}{T} \sum_{T \in t_{0}-t} D\left(A T M_{i}, T\right) \\
D\left(A T M_{i}, T\right)=\left\lceil U\left(A T M_{i}, T\right)\right\rceil-W\left(A T M_{i}, T\right)+U_{C}
\end{gathered}
$$

here, $S_{0}(A T M)$ is the initial stocked cash, $U\left(A T M_{i}, T\right) \in L$ is the upload applied to date $T, D\left(A T M_{i}, T\right)$ is cash's daily balance at date $T, W\left(A T M_{i}, T\right)$ is cash withdrawn at date $T$ and $U_{c}$ is the extra refill at date $T$ to handle out-of-service situation.

This article utilizes IT2FNN as mean to find an optimal specifications subset $K$. The general idea behind use of IT2FNN is that of allowing the network to map the relationships between various factors affecting the cash withdrawal and the actual cash withdrawal. Nowadays; fuzzy neural network controllers that can combine the advantages of fuzzy logic controllers and neural networks have been widely applied in many real word applications [10-13]. One way to build IT2FNN is to fuzzify a conventional neural network. A fuzzy neuron is basically similar to an artificial neuron except that it has the ability to process fuzzy information.

Type-2 fuzzy logic system are characterized by fuzzy IF-THEN rules but the membership functions are three dimensional and include a Footprint of Uncertainty (FOU)[14]. FOU provides additional degrees of freedom that make it possible to directly model and handle uncertainties associated with the inputs and outputs of real system. Type-2 fuzzy rules are more complex than type- 1 fuzzy rules because of their use of type-2 fuzzy sets in antecedent or consequent parts. Therefore, most T2FNN research is only concerned with interval type-2 fuzzy systems. The complete IT2FNN theory can be found in [11], [12].

Fig. 1 shows the employed network structure, which has a total of four layers. This network realized an interval type-2 fuzzy system whose rule has the form in equation 1 . Neural network is brought forwarded to generate a fuzzy inference system structure automatically for the input. Note that, when the algorithm is instanced, an initial population of rule specification sets is randomly generated. These specifications 
provide the set of condition and recharge target values required to build rules. Detailed mathematical functions of each layer are introduced as follows, and a more complete description for these layers can be found in [10], [13].

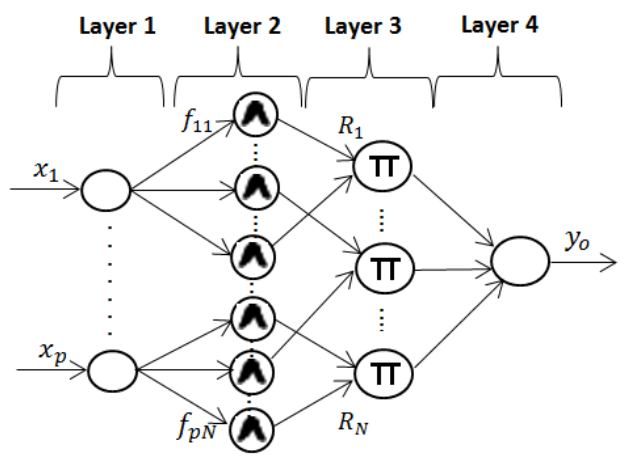

Fig. 1. Generic structure of IT2FNN

\section{A. Layer 1: Input Layer}

This layer represents a possible set of rule specification that is a point in $R$. The number of neurons in this layer depends upon: (a) the number of cash withdrawal factors $(p)$ and (b) way these factors are encoded. No weights to be adjusted in this case. The input variables, $x_{i}, i=1,2, \ldots, p$, are crisp values that are coded values of: (1) Calendar effects;(2) Month of the year; (3) Current availability of cash; (4) Average daily cash demand in the last week, and (5) ATM location (Lobby- Shopping center- plazas - tourist location, and in the bank). The net input and output of the $i^{\text {th }}$ node are represented as:

$$
n_{i}^{1}=x_{i}, \quad y_{i}^{1}=n_{i}^{1}, \quad i=1,2, \ldots, p
$$

In this scenario, given the cash limit $M$, conditions on both current availability of cash $S$ and cash upload $U$ are reported in Table I. While, conditions on calendar effects with respect to the current date are: (i) working day, (ii) weekday, (iii) holiday effect, (iv) salary day effect and (v) festival day effect. Once the necessary inputs (factors) are identified, it is relatively simple to train IT2FNN to form a non-linear model of the underlying forecasting system and then use this model to generalize new cases that are not part of the training data.

TABLE I: CASH STOCK AND UPLOAD STATEMENTS

\begin{tabular}{lll}
\hline & Cash stock level & Cash upload Level \\
\hline (i) & $S<0.2 M$ & $U=M$ \\
(ii) & $0.2 M \leq S<0.3 M$ & $U=0.67 *(M-S)$ \\
(iii) & $0.3 M \leq S<0.4 M$ & $U=0.5 *(M-S)$ \\
(iv) & $0.3 M \leq S<0.4 M$ & $U=0.33 *(M-S)$ \\
(v) & $0.4 M \leq S<0.8 M$ & $U=0.2 *(M-S)$ \\
(vi) & $0.8 M \leq S<0.9 M$ & $U=0.1 *(M-S)$ \\
(vii) & $S \geq 0.9 M$ & $U=$ \\
\hline \hline
\end{tabular}

\section{B. Layer 2: Fuzzification Layer}

In this layer, there are $N \times P$ nodes that perform the fuzzification operation, where $N$ is the number of fuzzy rules in the rule base. The net output of the node $i j$, performs an interval type -2 membership function of the fuzzy set is:

$$
\begin{gathered}
y_{i j}^{2}=\left\lfloor\underline{y}_{i j}^{2}, \bar{y}_{i j}^{2}\right\rfloor, j=1,2, \ldots, N, i=1, \ldots, p \\
\underline{y}_{i j}^{2}=\underline{f}_{i j}^{2}\left(y_{j}^{1}\right), \quad \bar{y}_{i j}^{2}=\bar{f}_{i j}^{2}\left(y_{j}^{1}\right) \\
\bar{f}_{i j}^{2}\left(y_{j}^{1}\right)= \begin{cases}N\left(m_{j 1}^{i}, \sigma_{j}^{i}, y_{j}\right) & y_{i}<m_{j 1}^{i} \\
1 & m_{j 1}^{i} \leq y_{i} \leq m_{j 2}^{i} \\
N\left(m_{j 2}^{i}, \sigma_{j}^{i}, y_{j}\right) & y_{i}>m_{j 2}^{i}\end{cases} \\
\underline{f}_{i j}^{2}\left(y_{j}^{1}\right)= \begin{cases}N\left(m_{j 2}^{i}, \sigma_{j}^{i}, y_{j}\right) & y_{i} \leq \frac{m_{j 1}^{i}+m_{j 2}^{i}}{2} \\
N\left(m_{j 1}^{i}, \sigma_{j}^{i}, y_{j}\right) & y_{i}>\frac{m_{j 1}^{i}+m_{j 2}^{i}}{2}\end{cases}
\end{gathered}
$$

in which, $f_{i j}^{2}, \bar{f}_{i j}^{2}$ denote the lower and upper membership functions' FOU. For the $i^{\text {th }}$ fuzzy set of the input variable $y$, a Gaussian primary membership function having a fixed standard deviation $\sigma$ and an uncertain mean that takes on values in $\left[m_{j 1}, m_{j 2}\right]$ is used because their center parameter is easily adaptable for many applications:

$$
N\left(m_{j}^{i}, \sigma_{j}^{i}, y_{j}\right)=\exp \left\{-\frac{1}{2}\left(\frac{y_{j}-m_{j}^{i}}{\sigma_{j}^{i}}\right)^{2}\right\}
$$

\section{Layer 3: Rule Layer}

Each node in this layer corresponds to one fuzzy rule in the rule base that performs the fuzzy algebraic product operation (fuzzy inference). The rules use the input membership values as weighting factors to determine their influence on the fuzzy output sets of the final output conclusion. The output of each node is a firing strength $F^{i}$ computed as:

$$
F^{i}=\left[\prod_{i=1}^{p} \underline{y}_{i j}^{2}, \prod_{i=1}^{p} \bar{y}_{i j}^{2}\right]=\left[y_{i}^{3}, \bar{y}_{i}^{3}\right]
$$

\section{Layer 4: Output Layer}

Node in this layer computes the output linguistic variable y using the type reduction and de-fuzzification processes. Because the output of layer 3 is type- 2 interval set, the COS (center of set) type- reduction method is adopted in which the left end point, $\underline{n}^{4}$, and the right end point, $\frac{-4}{n}$, of the type-reduced set can be obtained as :

$$
\begin{gathered}
\underline{n}^{4}=\frac{\sum_{i=1}^{N} a_{l, i} \bar{y}_{i}^{3}+\sum_{i=1}^{N} b_{l, i} \underline{y}_{i}^{3}}{\sum_{i=1}^{N} c_{l, i} \bar{y}_{i}^{3}+\sum_{i=1}^{N} d_{l, i} \underline{y}_{i}^{3}} \\
\bar{n}^{4}=\frac{\sum_{i=1}^{N} a_{r, i} y_{i}^{3}+\sum_{i=1}^{N} b_{r, i} \bar{y}_{i}^{3}}{\sum_{i=1}^{N} c_{r, i} \underline{y}_{i}^{3}+\sum_{i=1}^{N} d_{r, i} \bar{y}_{i}^{3}}
\end{gathered}
$$

The parameters $a_{l, i}, b_{l, i}, c_{r, i,}$ and $d_{r, i}$ can be computed in the 
forward-propagation derivatives process of the IT2FNN [14]. The defuzzified output of the node can be represented as:

$$
y_{O}^{4}=\frac{1}{2}\left(\underline{n}^{4}+n^{-4}\right)
$$

The learning procedure of the employed IT2FNN has two parts: in the first part the input patterns are propagated, both consequent factors and premise upload level variable are assumed to be fixed for the current cycle through the training set. In the second part, pack-propagation is used to modify the parameters. Herein, there is no need to determine IT2FNN structure in advance because the utilized model has on-line structure learning ability that makes it more suitable for handling time-varying systems than other IT2FNN systems, which adjust their parameters based on pre-trained and fixed structure ( i.e. has no structure learning and the number of rules is assigned a priori).

The utilized IT2FNN has two types of learning: structure and parameter learning [11][12]. In case of structure learning, the firing strength can serve as a rule generation criterion that is, for each piece of incoming factors $x=\left(x_{1}, x_{2}, \ldots x_{N}\right)$ finds $I$ such that:

$$
I=\arg \max _{1 \leq i \leq Q(t)} f_{c}^{i}(x), \quad f_{c}^{i}=\frac{1}{2}\left(\bar{f}^{i}+\underline{f}^{i}\right)
$$

where $Q(t)$ is the number of existing rules at time $t$. In this, a firing strength can be regarded as the degree to which an input data belongs to cluster (a rule corresponds to a cluster in the input space). Repeating the above process for every incoming piece of training data generates new rules, one after another, until the complete IT2FNN is finally constructed. Regarding parameter learning, the back propagation method is utilized to adjust the parameters of IT2FNN in order to minimize the error term in each layer. Readers looking for comprehensive details about back propagation implementation can refer to [15].

\section{Simulations AND VALidATion}

In this section, an application to the cash demand forecasting is investigated to demonstrate the effectiveness of the employed model. In parallel, the proposed forecaster is compared with state-of-the-art forecasting approaches

\section{A. Experimental Setup}

To test the potential of the proposed method to accurately forecast the cash demand in ATM network, a simulation environment for ATM network was designed. The activity of ATM network, which consists of 25 ATMs, was simulated. Daily, weekly and monthly seasonality along with long-term styles and special events or localized abrupt changes (holiday and festival effects) were used to imitate the customers' money withdrawal from ATMs that are characterized by different transaction volumes.

The realization of IT2FNN-based forecasting method was implemented using MATLAB ${ }^{\mathrm{TM}}$ programming environment with Simulink toolbox. Simulink is highly configurable and extendable simulation software supporting up-to-date features of the recent simulation research. Tests ran on Intel Xeon $2.66 \mathrm{GHz}$ machine with 4GB RAM equipped with operating system Windows XP professional platform. Each test was setup with IT2FNN's initial uncertain mean $m_{j}^{i}$ and standard deviation $\sigma_{j}^{i}$ for the new interval type-2 fuzzy set in input variable $x_{j}$ as $m_{j}^{i} \in\left[x_{j}-0.1, x_{j}+0.1\right], \quad \sigma_{j}^{i}=0.2$.

\section{B. Execution and Results}

Test group 1 is intended for comparing the results offered by the proposed methodology with historical weekly cash withdrawal actions as performed by human in the simulator for $\mathrm{ATM}_{1}$ that facing non stationary of cash demand. Results reported in Fig. 2 confirm that ATM cash management can improve by application of fuzzy neural network, leading to a lower amount of stocked cash. The average generalization forecast accuracy (per week) of the proposed method is about $97.72 \%$ while the minimum forecast accuracy is $94.15 \%$.

Validity of the results are confirmed by test group 2, aimed at testing rules found in the past when they are applied to the future period within an horizon of one month. Again, actual cash withdrawal is convergent to the upload strategy suggested by the IT2FNN-forecasting system as outlined in table II. This result is according to extreme heterogeneity of ATMs cash demand profiles in the simulator. Form table II, anyone can affirm that rules found in the training phase are consistent and valid when applied to the specified time period.

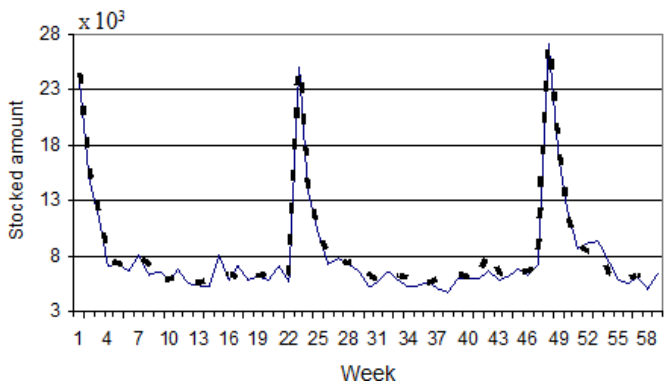

Fig. 2. Forecasting results for the analyzed data: actual values (solid line) and predicated values (dashed line).

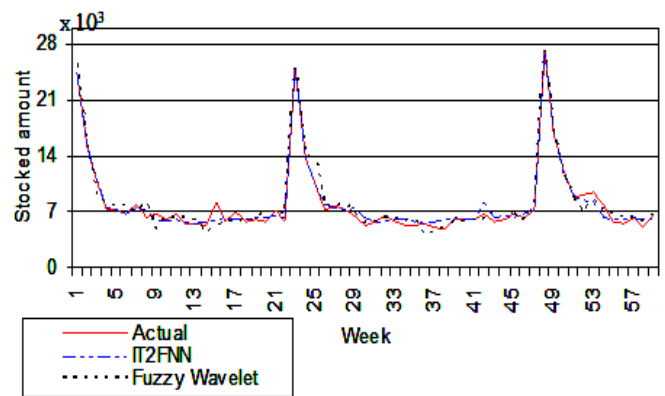

Fig. 3. Predictions comparison for different forecasting methods.

Test group 3 aimed at assessing the advantages in relying forecasting on fuzzy neural network instead of fuzzy logic based-time series predication approach established by the authors in [16]. Their method is based on the combination of wavelet analysis and fuzzy modeling to deal with time series process that has seasonal variations, long term and shot term fluctuations. 
TABLE II: AVERAGE WEEKLY STOCKED CASH $\left(\mathrm{X} 10^{3}\right)$ OF THE SUGGESTED RULES IN THE TESTING SET.

\begin{tabular}{|c|c|c|c|c|c|c|}
\hline \hline & \multicolumn{2}{|c|}{ ATM $_{1}$} & \multicolumn{2}{c|}{ ATM $_{14}$} & \multicolumn{2}{c|}{ ATM $_{22}$} \\
\cline { 2 - 7 } & A & P & A & P & A & P \\
\hline Week 1 & 49.17 & 50.1 & 19.96 & 20.3 & 6.62 & 7.1 \\
\hline Week 2 & 48.74 & 48.9 & 27.56 & 28.13 & 4.96 & 5.16 \\
\hline Week 3 & 159.24 & 160.5 & 19.73 & 19.8 & 6.01 & 6.42 \\
\hline Week 4 & 51.8 & 52.01 & 18.65 & 20.02 & 50.9 & 51.2 \\
\hline \hline
\end{tabular}

TABLE III: SUMMARY OF EXPERIMENTAL RESULT.

\begin{tabular}{|c|c|}
\hline \hline Forecasting Method & MFE \\
\hline IT2FNN & 6.566 \\
\hline Fuzzy Wavelet-based & 19.438 \\
\hline \hline
\end{tabular}

Fig. 3 displays the comparison between the two methods and their error (i.e. deviation from the actual withdrawal) indicating that the employed fuzzy neural network has a smaller divergence and has a better predication than that for time series with a $13 \%$ reduction in the MFE (mean forecast error) as seen in table III. This suggests that even with highly non-stationary of the underlying withdrawal process, the proposed model exhibits certain regularities and rules, which can be effectively modeled to give reasonable predictions. Furthermore, the ability to predict cash demand within reasonable accuracy of actual demand provides target for supply optimization well in time.

\section{CONCLUSION}

Optimizing cash in ATMs is difficult due to unpredictability of withdrawals. Therefore, finding the best match between cash stock and demand becomes crucial to improve. This paper presented a method based on IT2FNN aimed at searching optimal strategies to refill ATM cash stocks to meet non-stationary of cash demand. Differently from approaches based on predication of future cash demand, the proposed method is based on fuzzy rules regarding some factors that if met suggest to upload cash. Such a system will help the bank for proper and efficient cash management and can be scaled for all branches of a bank by incorporating historical data from these branches.

The performed simulation of ATM network's cash forecasting system showed good results and proved that this method is feasible in suggesting reliable rules able to improve historical cash management. However, there are other factors like festival period and market activities that positively influence cash demand and if these are quantified and included as influencing variables, the result will improve with lesser error. The system performs better than other systems based on time series. The future work will be directed on implementing coordinated route planning technique for reducing the ATM network's management costs and build an adaptive ATM cash management and support system.

\section{REFERENCES}

[1] R. Simutis, D. Dilijonas, L. Bastina, and J. Friman, "A Flexible Neural Network for ATM Cash Demand Forecasting," in Proc. 16th Int. Conf. on Computational intelligence, Man-Machine System and Cybernetics, Spain, Dec. 14-16, 2007, pp. 162-165

[2] D. Dilijonas and L. Bastina, "Retail Banking Optimization System Based on Multi-agents Technology," in Proc. 16th Int. Conf. on Computational intelligence, Man-Machine System and Cybernetics, Spain, Dec. 14-16, 2007, pp. 203-208.

[3] M. Wagner, "Forecasting Daily Demand in Cash Supply Chains," American Journal of Economics and Business Administration, vol. 2, no.4, pp. 377-383, 2010

[4] A. R. Brentnalla, M. J. Crowderb, and D. J. Handb, "Predictive-Sequential Forecasting System Development for Cash Machine Stocking," International Journal of Forecasting, vol. 26 pp. 764-776, 2010.

[5] I. Aydin, M. Karakose, and E. Akin, "The Prediction Algorithm Based on Fuzzy Logic Using Time Series Data Mining Method," World Academy of Science, Engineering and Technology, vol. 51, pp. 91-98, 2009.

[6] R. Simutis, D. Dilijonas, and L. Bastina, "Cash Demand Forecasting For ATM Using Neural Networks And Support Vector Regression Algorithms," in Proc. 20th EURO Mini Conference on Continuous Optimization and Knowledge-Based Technologies ((EurOPT-2008), May 20-23, Lithuania, 2008, pp. 416-421.

[7] S. D. Teddy and S. K. Ng, "Forecasting ATM Cash Demands Using a Local Learning Model of Cerebellar Associative Memory Network," International Journal of Forecasting, vol. 27, pp. 760-776, 2011.

[8] P. Kumar and E. Walia, "Cash Forecasting: An Introduction of Artificial Neural Networks in Finance," International Journal of Computer Sciences and Applications, vol. 3, no. 1, pp. 61-77, 2006.

[9] R. Armenise, C. Birtolo, E. Sangianantoni, and 1. Troiano, "A Generative Solution for ATM Cash Management," Int. Conf. of Soft Computing and Pattern Recognition, France, 7-10 Dec. 2010, pp. 349-356.

[10] C. F. Juang and R. B. Huang, "A Mamdani Recurrent Interval Type-2 Fuzzy Neural Network for Identification of Dynamic Systems with Measurement Noise," in Proceedings of the 18th IFAC World Congress, Italy, Aug. 28-Sep. 2, 2011, pp. 8975-8980.

[11] C. S. Chen and W. C. Lin, "Self-Adaptive Interval Type-2 Neural Fuzzy Network Control for PMLSM Drives," Expert Systems with Applications, vol. 38, pp. 14679-14689, 2011.

[12] C. F. Juang, C. F. Lu, and W. Tsao, "A Self Evolving Interval Type-2 Fuzzy Neural Network for Nonlinear System Identification," in Proceedings of the 17th IFAC World Congress, Korea, July 6-11, 2008 , pp. 7588-7593.

[13] L. Zhao, "Short-Term Traffic Flow Prediction Based on Interval Type-2 Fuzzy Neural Networks," in Proc. LSMS /ICSEE2010, Springer, pp. 230-237, 2010.

[14] J. M. Mendel, "Computing Derivatives in Interval Type-2 Fuzzy Logic System," IEEE Trans. on Fuzzy Systems, vol. 10, no. 2, pp. 117-127, 2004.

[15] C. H. Lee, J. L. Hong, Y. C. Lin, and W. Y. Lai, "Type-2 Neural Network Systems and Learning," Int. Journal of Computational Cognition, vol. 1, no. 4, pp. 79-90, 2003.

[16] Y. Chena, B. Yanga, and J. Donga, "Time-Series Prediction Using a Local Linear Wavelet Neural Network," Neurocomputing, vol. 69, pp. 449-465, 2006.

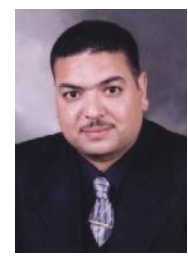

Saad M. Darwish received the B.Sc. degree in statistics and computer science from the faculty of science, Alexandria University, Egypt in 1995. He held the M.Sc. degree in information technology from the Institute of Graduate Studies and Research (IGSR), Department of Information Technology, University of Alexandria in 2002. He received his Ph.D. degree from the Alexandria University for a thesis in image mining and image description technologies. His research and professional interests include image processing, web engineering, security technologies and database management. He has published in journals and conferences and severed as TPC of a number of international conferences. Since Feb. 2012, he has been an Associate Professor in the department of information technology, IGSR. 